Journal for ImmunoTherapy of Cancer

\section{Mobilization of pre-existing polyclonal $T$ cells specific to neoantigens but not self-antigens during treatment of a patient with melanoma with bempegaldesleukin and nivolumab}

To cite: Veatch JR, Singhi N, Jesernig B, et al. Mobilization of pre-existing polyclonal $\mathrm{T}$ cells specific to neoantigens but not self-antigens during treatment of a patient with melanoma with bempegaldesleukin and nivolumab. Journal for ImmunoTherapy of Cancer 2020;8:e01591. doi:10.1136/ jitc-2020-001591

- Additional material is published online only. To view, please visit the journal online (http://dx.doi.org/10.1136/jitc2020-001591).

Accepted 09 November 2020

Check for updates

(c) Author(s) (or their employer(s)) 2020. Re-use permitted under CC BY-NC. No commercial re-use. See rights and permissions. Published by BMJ.

${ }^{1}$ Clinical Research Division, Fred Hutchinson Cancer Research Center, Seattle, Washington, USA ${ }^{2}$ Nektar Therapeutics, San Francisco, California, USA

${ }^{3}$ Department of Medical Oncology, University of Washington, Seattle, Washington, USA

Correspondence to Dr Joshua R Veatch; jveatch@fhcrc.org

\section{ABSTRACT}

T cells that recognize self-antigens and mutated neoantigens are thought to mediate antitumor activity of immune checkpoint blockade (ICB) in melanoma. Few studies have analyzed self and neoantigen-specific T cell responses in patients responding to ICB. Here, we report a patient with metastatic melanoma who had a durable clinical response after treatment with the programmed cell death protein 1 inhibitor, nivolumab, combined with the first-in-class CD122-preferential interleukin-2 pathway agonist, bempegaldesleukin (BEMPEG, NKTR214). We used a combination of antigen-specific $T$ cell expansion and measurement of interferon- $\gamma$ secretion to identify multiple $\mathrm{CD}^{+}{ }^{+}$and $\mathrm{CD}^{+}{ }^{+} \mathrm{T}$ cell clones specific for neoantigens, lineage-specific antigens and cancer testis antigens in blood and tumor from this patient prior to and after therapy. Polyclonal $\mathrm{CD} 4^{+}$and $\mathrm{CD}^{+} \mathrm{T}$ cells specific to multiple neoantigens but not self-antigens were highly enriched in pretreatment tumor compared with peripheral blood. Neoantigen, but not self-antigen-specific T cell clones expanded in frequency in the blood during successful treatment. There was evidence of dramatic immune infiltration into the tumor on treatment, and a modest increase in the relative frequency of intratumoral neoantigen-specific $T$ cells. These observations suggest that diverse $\mathrm{CD}^{+}$and $\mathrm{CD} 4^{+} \mathrm{T}$ cell clones specific for neoantigens present in tumor before treatment had a greater role in immune tumor rejection as compared with self-antigen-specific T cells in this patient. Trial registration number: NCT02983045.

\section{INTRODUCTION}

$\mathrm{T}$ cells that recognize neoantigens resulting from cancer-specific mutations are often detected in patients with melanoma and other cancers and are thought to contribute to effective antitumor immunity. ${ }^{1-3}$ In melanoma, $\mathrm{T}$ cells can also recognize antigens from melanocyte lineage specific proteins and re-expressed cancer-testis antigens. ${ }^{4}$

$\mathrm{T}$ cell receptor (TCR) sequencing allows for precise quantitation of individual $\mathrm{T}$ cell clonotypes within clinical samples, but identifying which $\mathrm{T}$ cell clones are specific for individual tumor antigens is challenging because such cells are usually present in low frequency in the blood $^{5-7}$ and in tumors. ${ }^{8}$ Rare $\mathrm{T}$ cells can be expanded from the blood with antigen stimulation, ${ }^{9}$ allowing the use of TCR sequencing to detect clonotypes that increase in frequency following stimulation with peptide antigen to identify specific $\mathrm{T}$ cells. ${ }^{510}$ Here, we use peptide stimulation and TCR sequencing to identify clonally diverse $\mathrm{CD}^{+}$and $\mathrm{CD} 4^{+} \mathrm{T}$ cell responses to multiple neoantigens and self-antigens and study their dynamics and localization during successful treatment of a patient with metastatic melanoma.

\section{METHODS}

\section{Research samples}

Research blood samples were collected at day -13, 42, 63 and 455 on Fred Hutchinson Cancer Research Center (FHCRC) protocol 1765 and tumor biopsies were obtained on the clinical trial protocol 6 days before and 17 days after initiation of treatment.

\section{Whole exome sequencing, RNA sequencing, neoantigen prediction}

Whole exome sequencing (WES) of pretreatment tumor biopsy and peripheral blood mononuclear cell (PBMC) was performed and single nucleotide variants called by both Mutect ${ }^{11}$ and Strelka ${ }^{12}$ were filtered for a variant allele frequency (VAF) $>0.2$ (online supplemental figure S1). The 45 mutations with the highest level of mRNA expression (TPM >12) were selected for screening (online supplemental table S1). RNA 
sequencing was performed as described previously and in the online supplemental methods. ${ }^{13}$

\section{T cell culture and TCRV $\beta$ sequencing}

PBMC were cultured for 13 days in the presence of $2 \mu \mathrm{g} /$ $\mathrm{mL}$ peptide encompassing each neoantigen (27-mer with mutated amino acid at position 14, $80 \%$ pure, Elim Biopharma) or self-antigen (Peptivator, Milltenyi) in the presence of interleukin (IL)-21, IL-15, and IL-7 (Peprotech) as described previously. ${ }^{13}{ }^{14}$ Reactivity of cultures with individual peptides was assayed by interferon (IFN) $-\gamma$ ELISpot assay $(10 \mu \mathrm{g} / \mathrm{mL}$ peptide, ELISpot Pro human IFN- $\gamma$, Mabtech) ${ }^{13}{ }^{14}$ IFN- $\gamma$ secretion assay used the IFN- $\gamma$ secretion kit (Milltenyi). Sorted IFN- $\gamma$-positive cells were expanded in 96-well plates with allogeneic irradiated PBMC, phytohemagglutinin and IL-2 for 14-21 days as described. $^{14}$

TCRV $\beta$ sequencing was performed on DNA samples using the human TCRB kit from Adaptive Biotechnologies, and data were analyzed using Adaptive software. Frequencies of TCR clonotypes were determined by amino acid sequence (online supplemental table S2). Clonotypes identified in the T cell cultures were presumed antigen specific if they met the following criteria: (1) expansion to $>5$ templates with specific stimulation and at least 10 -fold enrichment in frequency over any other stimulation and (2) greater frequency of antigen-dependent IFN- $\gamma$ secreting cells than either prestimulation sample or irrelevant antigen control culture, with at least five templates observed in the IFN- $\gamma$-enriched sample. CDR3 consensus sequences were generated using weblogo ${ }^{15}$ ( weblogo.berkeley.edu).

\section{STATISTICS}

Data were analyzed using GraphPad Prism software. ELISpot data were analyzed using unpaired t-tests without correction for multiple comparisons. Relative enrichment of TCRV $\beta$ templates was performed using Fisher's exact test.

\section{RESULTS}

\section{Clinical presentation}

A man aged 21 years presented with a lung nodule that was determined by core needle biopsy to be metastatic melanoma, BRAF V600E mutated. Staging showed multiple lung nodules and mediastinal lymphadenopathy, without a detectable primary skin lesion. The patient was enrolled in a phase I clinical trial combining nivolumab $360 \mathrm{mg}$ and bempegaldesleukin $0.06 \mathrm{mg} / \mathrm{kg}^{16}$ intravenously every 3 weeks. A CT scan at 4.5 months showed a partial response by RECIST V.1.1 (figure 1A,B) and positron emission tomography showed no Fluorodeoxyglucose (FDG) avid disease after 13 months of therapy. The patient developed vitiligo 1 year following initiation of treatment and bempegaldesleukin was discontinued after 15 months due to recurrent grade 1 fatigue and myalgias. Nivolumab was discontinued after 2 years and the patient has not progressed after $>3$ years (figure 1A).

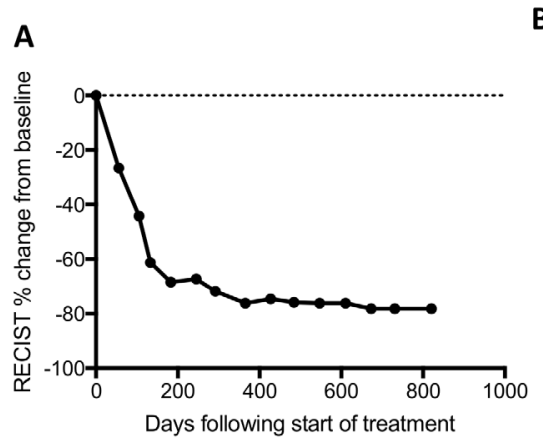

B

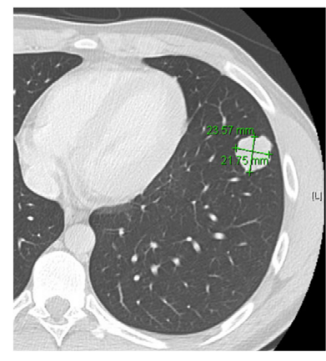

Pre-treatment

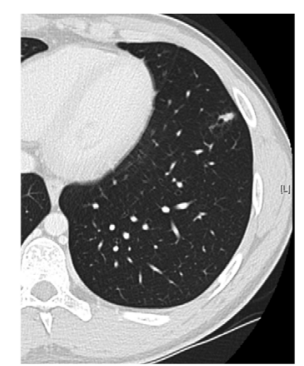

18 weeks into treatment

C

D
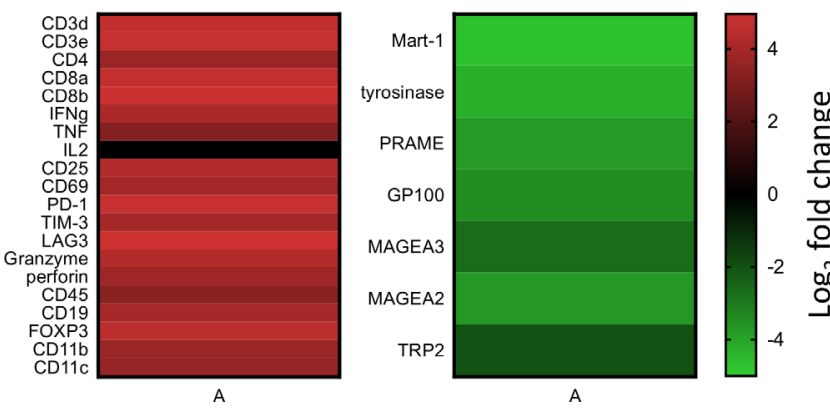

Figure 1 Treatment response and transcriptome analysis. (A) RECIST clinical disease burden following initiation of treatment. (B) Lung nodule on CT scan that underwent needle biopsy at day 19 of treatment. (C) Log $_{2}$ fold change of immune-related

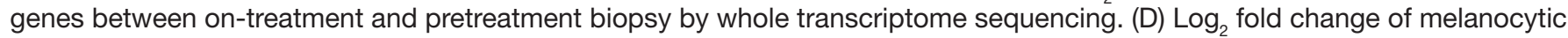
lineage-specific and cancer-testis antigens between on-treatment and pre-treatment tumor biopsy. 
A

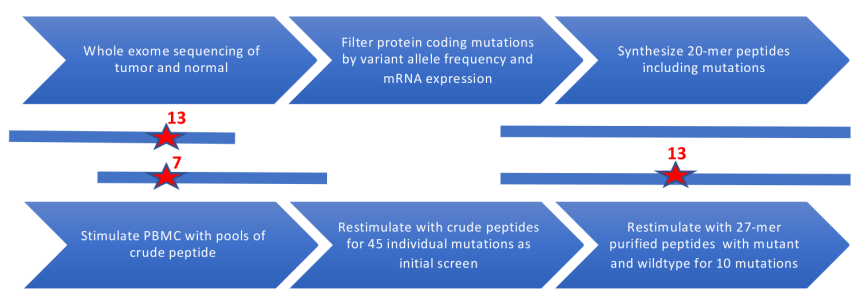

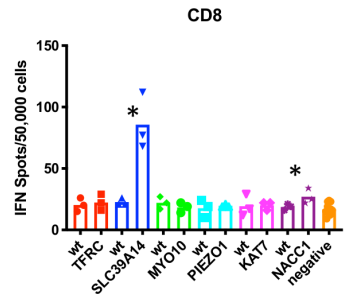

C

CD4

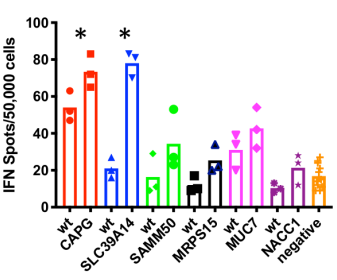

E.

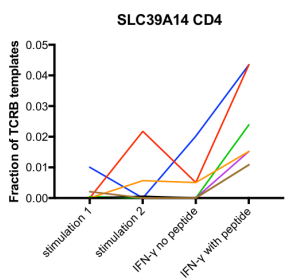

D.
2. Antigen specific IFN- $\gamma$ secretion
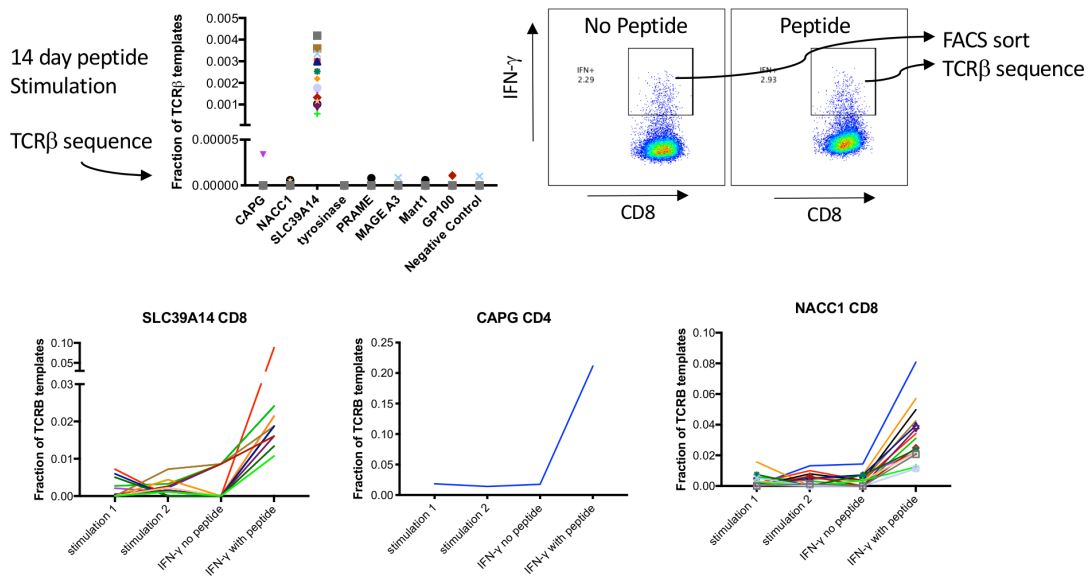

Figure 2 Identification of neoantigen-specific T cell clones. (A) Schematic of approach for identifying $T$ cell responses to neoantigens. (B-C) Peripheral blood mononuclear cells (PBMCs) were stimulated with pools of crude peptides and then magnetically enriched into $\mathrm{CD}^{+}$or $\mathrm{CD} 4^{+}$T cell subsets. $\mathrm{CD} 8^{+}(\mathrm{B})$ and $\mathrm{CD} 4^{+}(\mathrm{C}) \mathrm{T}$ cells were restimulated with single purified wild-type or mutant peptides and interferon (IFN)- $\gamma$ secretion was quantitated by ELISpot. (D) PBMCs were stimulated with purified peptides containing mutations and TCRV $\beta$ clonotypes were quantitated by sequencing (left panel). IFN- $\gamma$ secreting cells were identified following stimulation with peptide or no peptide (control) and sorted by fluorescence activated cell sorting (FACS) and subjected to TCRV $\beta$ sequencing. (E) Multiple CD4 ${ }^{+}$and $C D 8^{+}$clonotypes were enriched after IFN- $\gamma$ secretion after stimulation with the peptide containing the mutation in SLC39A14, CAPG or NACC1.

\section{Tumor regression is accompanied by an immune cell transcriptional signature}

Whole transcriptome sequencing performed on pretreatment and on-treatment tumor tissue from a lung lesion that subsequently completely regressed showed a $>10$ fold increase in expression of multiple genes annotated as immune response genes (3.47-fold enrichment, $\mathrm{p}=1.3 \mathrm{e}-81$ ), including those reflecting $\mathrm{T}$ cell infiltration (CD3, CD4 and CD8) and activation (IFN- $\gamma$, CD69, CD25, programmed cell death protein 1, Lag-3, Tim-3, granzyme, perforin and a decrease in lineage-specific melanocyte proteins tyrosinase, GP100, Mart1 and TRP2 and cancer testis antigens Mage A3, Mage A2 and PRAME (figure 1C,D, online supplemental table S2), consistent with nivolumab and bempegaldesleukin resulting in rapid immune infiltration and tumor regression. ${ }^{1617}$

\section{Identification of immunogenic neoantigens}

The oncogenic BRAF V600E mutation and 279 additional single nucleotide variants were identified as candidate neoantigens by WES of pretreatment tumor and germline DNA (online supplemental table S1). T cell responses were evaluated to 45 candidates with VAF $>0.2$ and expression $>12$ TPM. PBMCs were stimulated with a single pool of two 20 amino acid peptides encompassing each of the mutations and reactivity evaluated by IFN- $\gamma$ ELISpot (figure 2A). Following stimulation and prior to ELISpot assay, $\mathrm{CD} 8^{+} \mathrm{T}$ cells were enriched by positive immunomagnetic selection and analyzed separately from $\mathrm{CD} 8^{+}$ depleted PBMC. Candidate mutations that showed qualitative responses over baseline with crude mutated peptides (online supplemental figure S2A-C) were tested for IFN- $\gamma$ production in response to $80 \%$ purified 27 -mer peptides corresponding to the mutant and wild-type sequences in a confirmatory assay. We identified $\mathrm{CD} 8^{+} \mathrm{T}$ cell responses to mutations in SLC39A14 ( $\mathrm{p}<0.0001$ vs negative control, $\mathrm{p}=0.0096$ vs wild-type) and to NACC1 $(\mathrm{p}=0.0075$ vs negative control, $\mathrm{p}=0.09$ vs wild-type) (figure $2 \mathrm{~B}$ ). We also identified $\mathrm{CD} 4^{+} \mathrm{T}$ cell responses to the same mutation in SLC39A14 ( $p<0.0001$ vs negative control and $p=0.0004$ vs wild-type) and a mutation in CAPG ( $<<0.0001$ vs negative control and $\mathrm{p}=0.052$ vs the wild-type peptide, figure $2 \mathrm{C}$ ).

\section{Identification of neoantigen-specific TCR clonotypes}

We then used TCRV $\beta$ deep sequencing of PBMC that had been stimulated with individual peptides to identify putative antigen-specific $\mathrm{T}$ cell clonotypes that expand to one antigen and not others. ${ }^{10}$ TCR seq is robust in this assay to identify TCR clones present at high enough frequencies 
to be evenly distributed across parallel cultures. A limitation with this method is that clones present in only one culture could expand non-specifically. Indeed, it was common for some $\mathrm{T}$ cell clones to expand without antigen-specific stimulation in one but not in multiple other parallel cultures (online supplemental figure S3). As a second method of establishing antigen specificity, we tested whether restimulating the cultures with peptide and selecting IFN- $\gamma$ secreting $T$ cells by fluorescence activated cell sorting would further enrich antigen-specific cells (figure 2D) but not non-specifically expanded cells. Many T cells in these cultures secreted IFN- $\gamma$ even without antigen restimulation (figure 2D), therefore $\mathrm{CD}^{+}$and $\mathrm{CD}^{+}$IFN- $\gamma$ secreting cells were also sorted following control stimulations without peptide. With this combination of assays, we assigned the TCRV $\beta$ of putative antigen-specific cells to be those that 1 ) expanded with one antigen and no other antigens and 2) were enriched in IFN- $\gamma$ secreting cells with specific antigen stimulation to a greater degree than with control stimulation without antigen.

We then applied these methods to identifying putative neoantigen-specific TCRV $\beta$ clones in stimulations of post-treatment PBMC in separate cultures with purified peptides containing the mutations in CAPG, SLC39A14 and NACC1. We identified $16 \mathrm{CD}^{+}$clonotypes specific for SLC39A14, $8 \mathrm{CD} 4^{+}$clonotypes specific for SLC39A14, $16 \mathrm{CD}^{+}$clonotypes specific for NACC1 and a single $\mathrm{CD}^{+}$clonotype specific for CAPG (figure 2E, online supplemental table S3). Many of these TCR clonotypes were expanded in only one out of two independent stimulations of 5 million PBMC (seven $\mathrm{CD}^{+}$clones and all $\mathrm{CD}_{4}^{+}$clones specific for SLC39A14, and nine CD8 ${ }^{+}$ clones specific for NACC1, figure 2E) indicating the low frequency of many of these clonotypes capable of expanding with peptide stimulation.

\section{Isolation of $\mathrm{T}$ cell lines and confirmation of neoantigen specificity}

To confirm specificity, neoantigen-reactive $\mathrm{T}$ cell lines were obtained by expanding small numbers (3-30) of IFN- $\gamma$ secreting $\mathrm{T}$ cells after stimulation with mutated NACC1 and SLC39A14 peptides. T cell lines were then restimulated with peptide to identify those containing $>90 \%$ antigen-specific cells (figure 3A). TCRV $\beta$ deep sequencing of these lines was performed and demonstrated that many were highly oligoclonal (figure 3B-D). TCRV $\beta$ clonotypes present at a level of $>10 \%$ in these

A

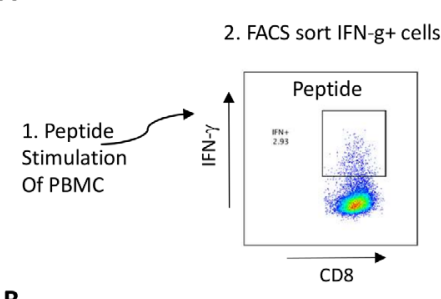

B

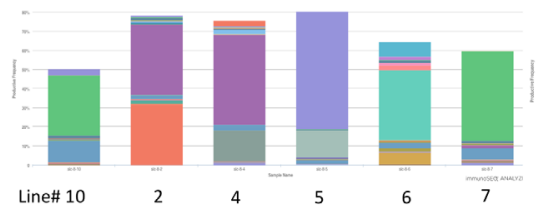

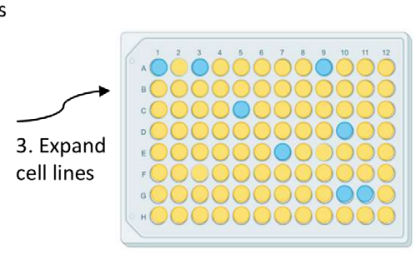

C

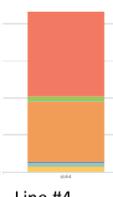

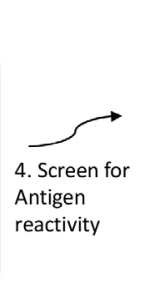

D
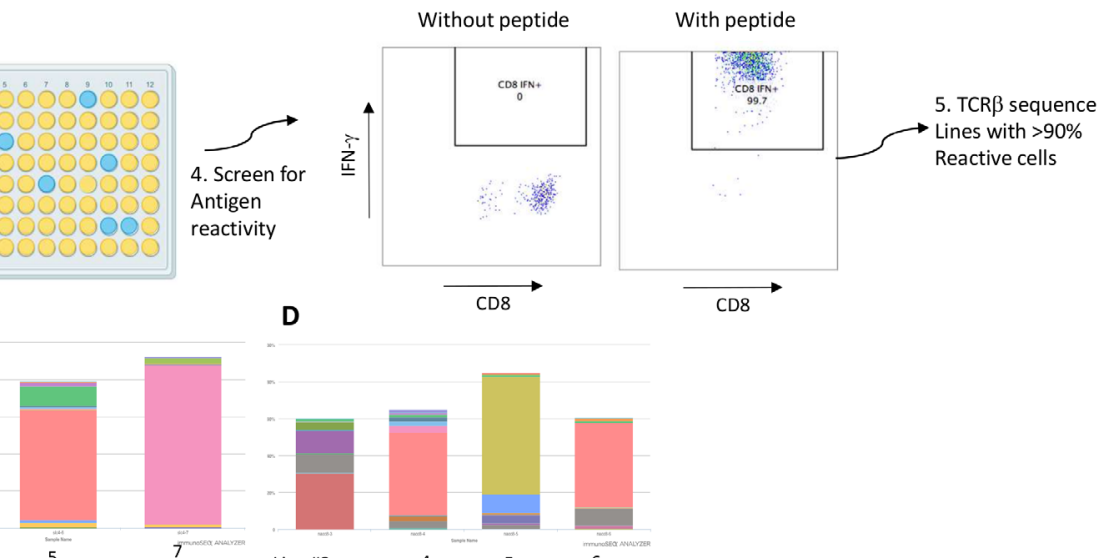

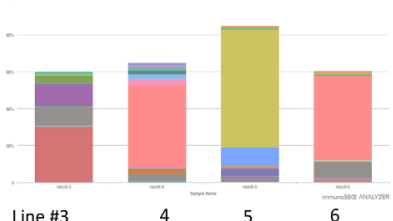

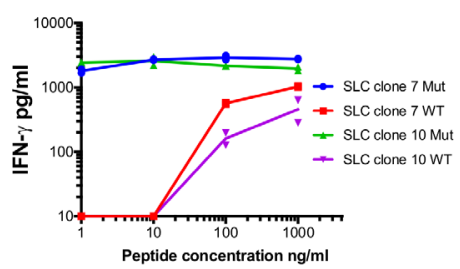

$\mathbf{F}$

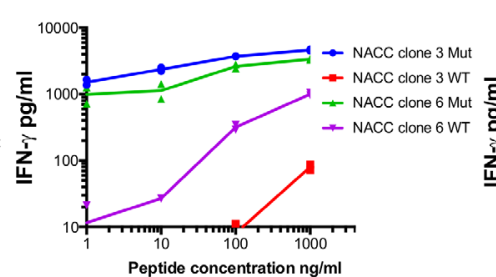

G

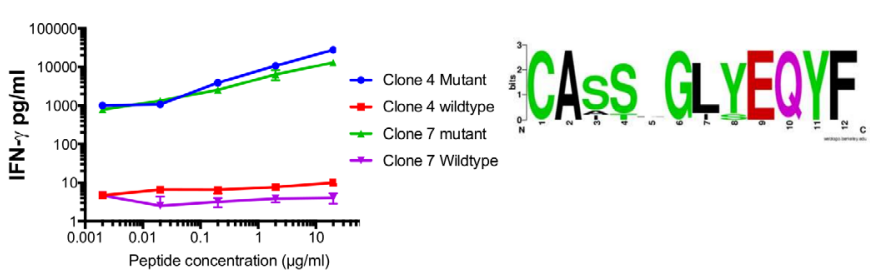

Figure 3 Isolation of patient-derived neoantigen-specific CD8 and CD4 T cells. (A) Schematic of strategy for isolating neoantigen-specific T cells: stimulation of peripheral blood mononuclear cell (PBMC) with peptides followed by isolation of interferon (IFN)- $\gamma$ secreting cells, expansion of T cell lines and screening of T cell lines for antigen specificity and TCRV $\beta$ sequencing of reactive $T$ cell lines. (B-D) Clonotype analysis of six reactive CD8 ${ }^{+} \mathrm{T}$ cell lines with each color representing an individual clone (B) and three CD4 ${ }^{+}$Tcell lines (C) specific for mutation in SLC39A14 and four CD8 ${ }^{+}$Tcell lines specific for a mutation in NACC1 (D). (E-G) Expanded CD8 ${ }^{+}$T cell lines were incubated with different concentrations of mutant and wild-type peptides containing mutations in SLC39A14 (E) and NACC1 (F) and CD4 ${ }^{+}$Tcell lines were incubated with peptides containing mutant and wild-type SLC39A14. (G) Sequences and IFN- $\gamma$ secretion was measured by ELISA assay. (H) Consensus sequence of nine NACC1-specific TCRV $\beta$ CDR3 amino acid sequences of length 12 were generated using weblogo. 
cultures were presumed to be antigen specific, which identified four $\mathrm{CD} 4^{+} \mathrm{T}$ cell clones specific for SLC39A14, seven $\mathrm{CD}^{+}{ }^{+}$cell clones specific for SLC39A14 and four CD8 ${ }^{+}$ Tcell clones specific for NACC1 (figure 3B-D, online supplemental table S3). Five of these 15 TCR clonotypes from this analysis overlapped with those previously identified, but multiple additional clonotypes were identified in cultures for each antigen (online supplemental table S3). To assess neoantigen specificity, oligoclonal $\mathrm{CD}^{+} \mathrm{T}$ cell lines derived by stimulation with SLC39A14 and NACC1 mutant peptides and $\mathrm{CD} 4^{+} \mathrm{T}$ cell lines derived by stimulation with the SLC39A14 mutant peptide were tested for reactivity with the mutant and wild-type peptides at various concentrations. Two different $\mathrm{CD} 8^{+} \mathrm{T}$ cell lines showed preferential activation with a 9-mer peptide containing the SLC39A14 mutation predicted to bind HLA-A:02, relative to the corresponding wild-type peptide (figure 3E), and two different $\mathrm{CD} 8^{+} \mathrm{T}$ cell lines reacted preferentially with a 10-mer mutated NACC1 mutant peptide predicted to bind HLA B15 (figure 3F). Similarly, two different $\mathrm{CD} 4^{+} \mathrm{T}$ cell lines were reactive with a 27 -mer peptide containing the mutation in SLC39A14 and not the wild-type peptide (figure $3 \mathrm{G}$ ). There was sequence similarity between TCRV $\beta$ hypervariable CDR3 sequences between independent clonotypes, such as nine different NACC1 mutation-specific CDR3 sequences of length 12 (figure $3 \mathrm{H}$ ). These data show that the expanded T cells were high avidity and neoantigen-specific.

\section{Neoantigen-specific $\mathrm{T}$ cell clones are present in the} pretreatment tumor and expand in the blood during treatment In total, there were 54 TCRV $\beta$ clonotypes identified to be neoantigen-specific (online supplemental table S3). To determine the localization of neoantigen-specific $\mathrm{T}$ cell clones during treatment, we performed TCRV $\beta$ sequencing of blood and tumor samples and found that 32 of 54 neoantigen-specific clones were detectable in the pretreatment tumor, which in total made up $0.71 \%$ of the TCRV $\beta$ templates in the pretreatment tumor (figure 4A, online supplemental table S2). In contrast, only four of these clones were detected in unstimulated pretreatment PBMC, making up $0.005 \%$ of TCRV $\beta$ sequences in the PBMC (150-fold enrichment in tumor, $\mathrm{p}<0.0001)$. Furthermore, only six of these clones could be detected in pretreatment PBMC after peptide stimulation of 5 million PBMC (online supplemental table S3).

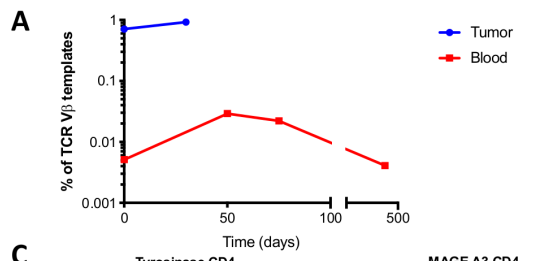

C
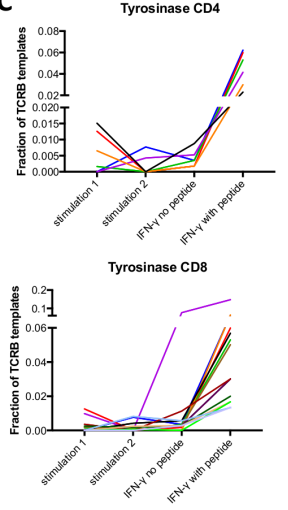

GP100 CD4

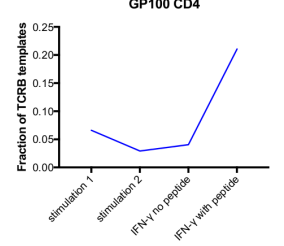

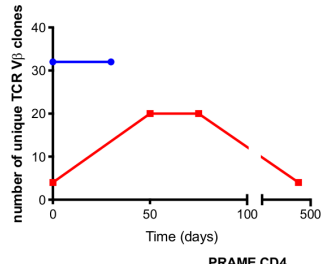

PRAME CD4
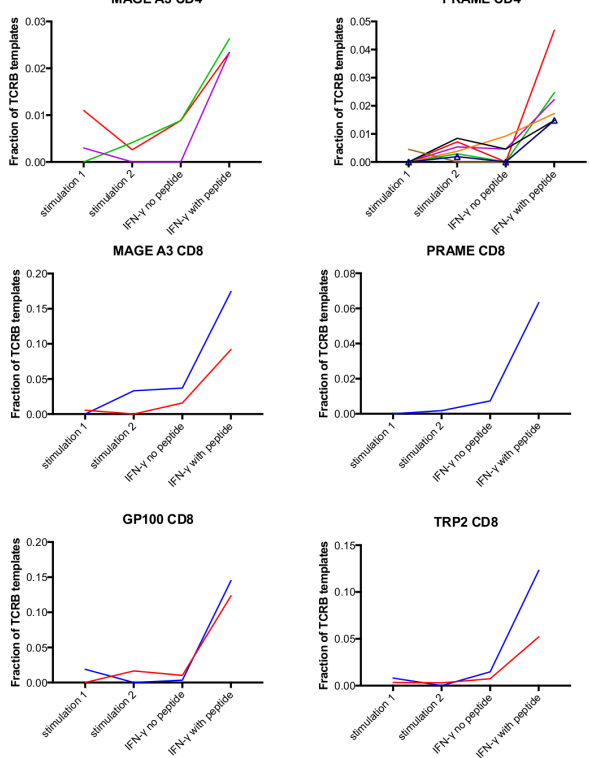

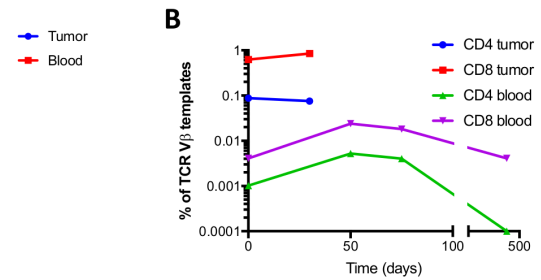

D

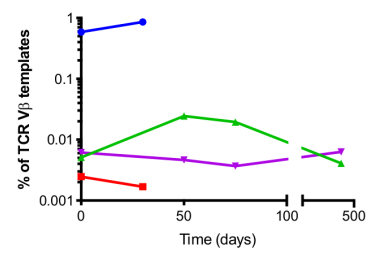

$\rightarrow$ Tumor-neoantigen

- Tumor-self antigen

- Blood-neoantigen

- Blood-self antigen (A-B) Cumulative frequency (left) or number of distinct neoantigen-specific TCRV $\beta$ clones (right) in tumor and blood samples at the indicated timepoints following treatment for total clones (A) and separated into CD $4^{+}$and $C D 8^{+}$clones (B). (C) Peripheral blood mononuclear cell (PBMC) was stimulated with purified peptides containing mutations or peptide pools of self-antigens and TCRV $\beta$ clonotypes were quantitated by sequencing in two independent replicates. These cultures were restimulated with peptides or no peptide controls, interferon (IFN)- $\gamma$ secreting cells were sorted by fluorescence activated cell sorting (FACS) and TCRV $\beta$ clonotypes were quantitated by sequencing. The frequency of putative antigen-specific clones is shown for $\mathrm{CD}^{+}$and $\mathrm{CD}^{+} \mathrm{T}$ cells for the indicated antigens. (D) Cumulative frequency (top panel) and number (bottom panel) of distinct neoantigen and self-antigen-specific TCRV $\beta$ clones in tumor and blood samples following treatment. 
Neoantigen-specific T cell clones expanded in frequency in the peripheral blood in the first 50 days following treatment (fivefold, $\mathrm{p}<0.0001$ ) and contracted in the blood 13 months after treatment (fivefold, $\mathrm{p}<0.0001$, figure $4 \mathrm{~A}$ ). RNA sequencing of a tumor sample after treatment demonstrated a prominent increase $\mathrm{T}$ cell transcripts (figure 1C), and the cumulative frequency of intratumoral neoantigen-specific TCRV $\beta$ increased modestly $(30 \%, \mathrm{p}<0.0001$, figure $4 \mathrm{~A})$, although the overall clonal diversity was similar prior to and on treatment (online supplemental figure S4). The findings that neoantigenspecific $\mathrm{T}$ cell clones were enriched in the tumor and expanded in the blood held true for neoantigen-specific $\mathrm{CD}^{+} \mathrm{T}$ cells $(\mathrm{p}<0.0001$ for enrichment in tumor, expansion in the blood following treatment and contraction following tumor regression) and for neoantigen-specific $\mathrm{CD} 4^{+} \mathrm{T}$ cells $(\mathrm{p}<0.0001$ for enrichment in the tumor, $\mathrm{p}=0.1$ for expansion in the blood post-treatment and $p=0.01$ for contraction in the blood post-treatment, figure $4 \mathrm{~B}$ ). Taken together, these data support the methodology for identifying $\mathrm{CD} 4^{+}$and $\mathrm{CD} 8^{+} \mathrm{T}$ cells that recognize tumor neoantigens, and demonstrate such clones are present in pretreatment tumor, rare in the peripheral blood before treatment and transiently expand in the blood during treatment.

\section{Self-antigen-specific $T$ cell clones do not localize to the tumor or expand during treatment}

We also assessed localization and expansion of $\mathrm{T}$ cell clones specific for self-antigens using similar stimulation and IFN- $\gamma$ secretion assays with overlapping long peptide pools encompassing lineage-specific (Tyrosinase, GP100, Mart1, TRP2) and cancer testis (MAGE A3, PRAME) selfantigens that were expressed in the tumor by RNA-seq. We identified $\mathrm{CD}^{+}(\mathrm{n}=2)$ and $\mathrm{CD}^{+}(\mathrm{n}=1)$ clonotypes specific for GP100; $\mathrm{CD}^{+}(\mathrm{n}=3)$ and $\mathrm{CD}^{+}(\mathrm{n}=2)$ clonotypes specific for MAGE A3; $\mathrm{CD} 4^{+}(\mathrm{n}=7)$ and $\mathrm{CD}^{+}(\mathrm{n}=1)$ clonotypes specific for PRAME; $\mathrm{CD}^{+}(\mathrm{n}=2)$ clonotypes specific for TRP2 and $\mathrm{CD}^{+}(\mathrm{n}=6)$ and $\mathrm{CD}^{+}(\mathrm{n}=12)$ clonotypes specific for tyrosinase (figure 4C, online supplemental table S3). We then compared self-antigen and neoantigen-specific clonotypes identified by the same method from the same blood sample and found, in contrast to neoantigen-specific T cell clones, self-antigenspecific $\mathrm{T}$ cell clones did not expand in the PBMC or localize to the tumor (figure 4D).

\section{DISCUSSION}

In this case report of effective immunotherapy in melanoma, we determined that diverse polyclonal $\mathrm{CD}^{+}$ and $\mathrm{CD} 4^{+}$responses to neoantigens were pre-existing in the patient, 150-fold enriched in tumor relative to blood and expanded 5-fold in the blood during the initial stages of treatment with nivolumab and bempegaldesleukin. In contrast, $\mathrm{T}$ cell responses to lineage-specific and cancer testis antigens did not localize to the tumor or expand with treatment. We cannot rule out contributions from $\mathrm{T}$ cell responses to self-antigens that were not identified in our assays, and the late clinical development of vitiligo suggested such responses were present at some point. ${ }^{18} 19$ However, our data are consistent with a prominent role for neoantigen relative to self-antigen-specific $\mathrm{T}$ cells in the clinical response. ${ }^{120}$ This could be due to central tolerance mechanisms that delete high avidity $\mathrm{T}$ cells recognizing self-antigens or due to tumor escape mechanisms that are selective for self-antigens. ${ }^{4} 2122$

$\mathrm{T}$ cell receptor sequencing of PBMC stimulated with specific peptides has been used as a method for identifying neoantigen-specific $\mathrm{T}$ cell clones, ${ }^{10}$ and we introduced a second step in which antigen-specific IFN- $\gamma$ secretion with antigen restimulation is used for further enrichment, followed by confirmatory analysis of recognition of mutant and wild-type peptides. We identified polyclonal $\mathrm{CD}^{+}$and $\mathrm{CD} 8^{+}$Tcell responses to multiple different neoantigens as well as lineage specific and cancer-testis antigens.

Pre-existing $\mathrm{T}$ cell responses to neoantigens were present in tumor but rare in the blood, suggesting challenges to isolating such $\mathrm{T}$ cells from the blood if they are not mobilized by effective treatment or enriched for a more tumor reactive subset. ${ }^{9}$ Indeed, the modest overlap in clonotypes identified by relative expansion versus limiting dilution cloning implies that both methods undersample the full set of neoantigen-specific clones, possibly a consequence of how rare these clones are in the periphery. Our RNA sequencing data suggested robust immune infiltration of the tumor 3 weeks post-treatment and a modest increase in the relative frequency of intratumoral neoantigen-specific cells.

In summary, we have described a method for systematically identifying neoantigen and self-antigen-specific $\mathrm{T}$ cell clones from the peripheral blood of a patient undergoing a clinical response to immunotherapy in melanoma, and highlighted a likely role for a pre-existing, polyclonal $\mathrm{CD}^{+}$and $\mathrm{CD} 8^{+}$Tcell response to tumor neoantigens, but not self-antigens, in the patient's clinical response.

Correction notice This paper has been updated since first published to correct author name 'Ernesto lacucci'.

Acknowledgements The authors would like to thank the patient and their family for participating in this research, and the Lembersky family for their generous support of this and other projects.

Contributors JRV, KGP, El, JZ and SR designed the experiments; JRV, NS and BJ performed the experiments; JV and SR analyzed the data; JRV, ST and SR wrote the paper.

Funding This work was funded by the NIH K12 grant CA076930-16A1 for JRV and generous support from the Bezos and Lembersky Families.

Competing interests JRV, BJ and SR have equity interest in Lyell Immunopharma, and $\mathrm{JZ}$ and EE are employees of Nektar therapeutics, and this paper discusses use of an investigational drug owned by Nektar therapeutics.

Patient consent for publication Obtained.

Ethics approval The patient signed informed consent for all protocols and all studies were approved by the institutional review board.

Provenance and peer review Not commissioned; externally peer reviewed. 
Data availability statement Data are available in a public, open access repository. Exome sequencing and RNA sequencing from this case will be submitted to the DbGap limited access database.

Supplemental material This content has been supplied by the author(s). It has not been vetted by BMJ Publishing Group Limited (BMJ) and may not have been peer-reviewed. Any opinions or recommendations discussed are solely those of the author(s) and are not endorsed by BMJ. BMJ disclaims all liability and responsibility arising from any reliance placed on the content. Where the content includes any translated material, BMJ does not warrant the accuracy and reliability of the translations (including but not limited to local regulations, clinical guidelines, terminology, drug names and drug dosages), and is not responsible for any error and/or omissions arising from translation and adaptation or otherwise.

Open access This is an open access article distributed in accordance with the Creative Commons Attribution Non Commercial (CC BY-NC 4.0) license, which permits others to distribute, remix, adapt, build upon this work non-commercially, and license their derivative works on different terms, provided the original work is properly cited, appropriate credit is given, any changes made indicated, and the use is non-commercial. See http://creativecommons.org/licenses/by-nc/4.0/.

\section{ORCID iD}

Joshua R Veatch http://orcid.org/0000-0002-6543-9255

\section{REFERENCES}

1 Schumacher TN, Schreiber RD. Neoantigens in cancer immunotherapy. Science 2015;348:69-74.

2 Lennerz V, Fatho M, Gentilini C, et al. The response of autologous T cells to a human melanoma is dominated by mutated neoantigens. Proc Natl Acad Sci U S A 2005;102:16013-8.

3 Gubin MM, Zhang X, Schuster $\mathrm{H}$, et al. Checkpoint blockade cancer immunotherapy targets tumour-specific mutant antigens. Nature 2014;515:577-81.

4 Gilboa E. The makings of a tumor rejection antigen. Immunity 1999;11:263-70.

5 Anagnostou V, Smith KN, Forde PM, et al. Evolution of neoantigen landscape during immune checkpoint blockade in non-small cell lung cancer. Cancer Discov 2017;7:264-76.

6 Balachandran VP, Łuksza M, Zhao JN, et al. Identification of unique neoantigen qualities in long-term survivors of pancreatic cancer. Nature 2017;551:512-6.

7 McGranahan N, Furness AJS, Rosenthal R, et al. Clonal neoantigens elicit $\mathrm{T}$ cell immunoreactivity and sensitivity to immune checkpoint blockade. Science 2016;351:1463-9.
8 Scheper W, Kelderman S, Fanchi LF, et al. Low and variable tumor reactivity of the intratumoral TCR repertoire in human cancers. Nat Med 2019;25:89-94.

9 Gros A, Parkhurst MR, Tran E, et al. Prospective identification of neoantigen-specific lymphocytes in the peripheral blood of melanoma patients. Nat Med 2016;22:433-8.

10 Danilova L, Anagnostou V, Caushi JX, et al. The mutation-associated neoantigen functional expansion of specific T cells (MANAFEST) assay: a sensitive platform for monitoring antitumor immunity. Cancer Immunol Res 2018;6:888-99.

11 Cibulskis K, Lawrence MS, Carter SL, et al. Sensitive detection of somatic point mutations in impure and heterogeneous cancer samples. Nat Biotechnol 2013;31:213-9.

12 Saunders CT, Wong WSW, Swamy S, et al. Strelka: accurate somatic small-variant calling from sequenced tumor-normal sample pairs. Bioinformatics 2012;28:1811-7.

13 Veatch JR, Lee SM, Fitzgibbon M, et al. Tumor-Infiltrating BRAFV600E-specific CD4+ T cells correlated with complete clinical response in melanoma. J Clin Invest 2018;128:1563-8.

14 Veatch JR, Jesernig BL, Kargl J, et al. Endogenous CD4 ${ }^{+} \mathrm{T}$ Cells Recognize Neoantigens in Lung Cancer Patients, Including Recurrent Oncogenic KRAS and ERBB2 (Her2) Driver Mutations. Cancer Immunol Res 2019;7:910-22.

15 Crooks GE, Hon G, Chandonia J-M, et al. Weblogo: a sequence logo generator. Genome Res 2004;14:1188-90.

16 Diab A, Tannir NM, Bentebibel S-E, et al. Bempegaldesleukin (NKTR214) plus nivolumab in patients with advanced solid tumors: phase I dose-escalation study of safety, efficacy, and immune activation (PIVOT-02). Cancer Discov 2020;10:1158-73.

17 Bentebibel S-E, Hurwitz ME, Bernatchez C, et al. A first-in-human study and biomarker analysis of NKTR-214, a novel IL2R $\beta \gamma$-biased cytokine, in patients with advanced or metastatic solid tumors. Cancer Discov 2019;9:711-21.

18 Yee C, Thompson JA, Roche P, et al. Melanocyte destruction after antigen-specific immunotherapy of melanoma: direct evidence of $T$ cell-mediated vitiligo. J Exp Med 2000;192:1637-44.

19 Le Gal FA, Avril MF, Bosq J, Lefebvre P, Deschemin J-C, et al. Direct evidence to support the role of antigen-specific CD8(+) T cells in melanoma-associated vitiligo. J Invest Dermatol 2001;117:1464-70.

20 Lauss M, Donia M, Harbst K, et al. Mutational and putative neoantigen load predict clinical benefit of adoptive $T$ cell therapy in melanoma. Nat Commun 2017;8:1738.

21 Kvistborg P, Shu CJ, Heemskerk B, et al. TIL therapy broadens the tumor-reactive CD8(+) T cell compartment in melanoma patients. Oncoimmunology 2012;1:409-18.

22 Effern M, Glodde N, Braun M, et al. Adoptive T cell therapy targeting different gene products reveals diverse and context-dependent immune evasion in melanoma. Immunity 2020;53:e9:564-80. 